\title{
Hábitos de sueño en el confinamiento por Covid-19 en una muestra de niños colombianos
}

\author{
Sleeping habits in confinement by Covid-19 \\ in a sample of Colombian children
}

\author{
Aldayr David Rosero Bolaños y Germán Emilio Ortega Enríquez
}

\author{
Universidad de Nariño ${ }^{1}$
}

Autor para correspondencia: Aldayr David Rosero Bolaños, davidbrs058@gmail.com.

\author{
RESUMEN
}

\begin{abstract}
La nueva enfermedad provocada por el virus SARS-COV-2 ha generado en el mundo un confinamiento cuyo fin es ralentizar el número de contagios y proteger así la vida de las personas; sin embargo, tal aislamiento y el cambio de hábitos han producido considerables efectos en su salud mental. Por consiguiente, el presente estudio tuvo como objetivo determinar los problemas en los hábitos de sueño y su relación con algunas características generadas por la reclusión en una muestra de 259 niños colombianos de 5 a 12 años de edad, residentes en la ciudad de Pasto (Colombia), a través de la aplicación a sus padres del cuestionario Children's Sleep Habits Questionnaire y de un cuestionario ad hoc para evaluar los cambios en las rutinas diarias, el uso de la tecnología y la emocionalidad en los niños. A este efecto, se hicieron análisis descriptivos de los instrumentos, una prueba de $\mathrm{X}^{2}$ para determinar la influencia de las variables de sexo y nivel socioeconómico, y pruebas de correlación rho de Spearman entre las escalas y subescalas de dichos instrumentos. Entre los resultados más relevantes se halló que $45.5 \%$ de los niños mostraban algunos problemas del sueño, siendo las parasomnias y la somnolencia diurna los de mayor prevalencia. Además, se encontraron correlaciones positivas entre los puntajes de las escalas, identificándose también relaciones entre el uso de la tecnología y los cambios emocionales con dificultades del sueño, por lo que se concluye que han ocurrido alteraciones en los hábitos de sueño debidos al confinamiento.
\end{abstract}

Palabras clave: Sueño; Hábitos; Confinamiento; Niños; Covid-19.

\begin{abstract}
The new disease caused by SARS-COV-2 has generated a process of global confinement to slow down the number of infections and protect people's lives. Isolation and changes in habits, however, have produced adverse effects on mental health. The present study aimed at determining problems in sleeping habits and their relationship with some conditions generated by confinement in a sample of Colombian children. The study included 259 parents of children aged 5 to 12 years from Pasto City. Data collected included the Children's Sleep Habits Questionnaire (CSHQ) and a questionnaire on daily routines, their use of digital technology, and changes in the children's emotionality. Descriptive analysis through the $X^{2}$ test determined the influence of sex and socioeconomic variables. Rho Spearman tests examined the correlation between the scales and subscales of the instruments. The most relevant results revealed that 45,5\% of children showed some sleep difficulties such as parasomnias and daytime sleepiness. Correlations between the scores (Rho $=0.46 p<0.05$ )
\end{abstract}

\footnotetext{
${ }^{1}$ Programa de Psicología, Colombia, CP 50002, teléfono: (02) 7244309, correos electrónicos: psicologia@udenar.edu.co, davidbrs058@ gmail.com y germoortega@hotmail.com.
} 
revealed associations among using digital technology and emotional changes with sleep difficulties. Thus results concluded that there are changes in sleep habits after the start of confinement.

Key words: Sleep; Habits; Confinement; Children; Covid-19.

Recibido: $10 / 08 / 2020$

Aceptado: 28/10/2020

$\mathrm{L}$ a crisis sanitaria generada por el nuevo Coronavirus SARS-CoV2, declarada como pandemia por la Organización Mundial de la Salud [OMS] (cf. Ferrer, 2020) a comienzos de marzo de 2020, ha llevado a los gobiernos y a la sociedad en general a tomar diferentes medidas cuyo fin es disminuir la tasa de contagios y de mortalidad en la población. Una de las estrategias más utilizadas y recomendadas por los expertos ha sido el aislamiento social, que consiste en evitar el contacto físico entre las personas en diferentes espacios, además de adoptar el confinamiento en casa como la manera ideal y más eficaz para ralentizar el proceso de contagio (Girona, 2020; González-Jaramillo et al., 2020).

No obstante, al ser una enfermedad nueva, el conocimiento que se tiene acerca de la misma es muy escaso, lo que ha sido un obstáculo para conocer la capacidad de transmisión, la sobrevivencia del virus activo en las superficies y en los seres humanos, los principales focos de infección y los posibles tratamientos farmacológicos, por mencionar algunos de los problemas más relevantes (Organización Panamericana de la Salud, 2020; Vargas, Acosta y Bernilla, 2020). Es por ello que las medidas que toman los Estados y las personas pueden no ser del todo confiables, lo que deja el confinamiento en casa y las nuevas vacunas como las únicas alternativas eficaces para disminuir el riesgo de contagio (Urzúa, Vera, Caqueo y Polanco, 2020).

Los diferentes estudios llevados a cabo en áreas como la psicología y ciencias afines se han centrado especialmente en los efectos del confinamiento en la población adulta y adolescente en problemas como la ansiedad, la depresión, los relativos a la condición física y el consumo de sustancias psicoactivas (Mera, Tabares, Montoya, Muñoz y Vélez, 2020). No obstante, las investigaciones sobre la población infantil son limitadas, evidenciando un gran vacío de conocimientos sobre los fenómenos generados en torno a la cuarentena que podría generar graves dificultades más adelante.

Aunado a lo anterior, son varios los autores que aducen un aumento en el número de casos de trastornos psicológicos, como ansiedad y depresión por las condiciones generadas por el confinamiento y su interacción con variables sociales, culturales, económicas e individuales, no estando los niños exentos de tales problemas de salud (Mazza et al., 2020; Zhou et al., 2020). Por tanto, existe la necesidad de investigar variables como los hábitos de sueño y descanso, los cuales tienen relaciones directas con la aparición y el desarrollo de diversas enfermedades mentales (Stanley et al., 2017).

Los patrones de sueño, su calidad y las conductas en torno al descanso han mostrado tener una considerable influencia en diferentes aspectos, que van desde el desarrollo adecuado de ciertas estructuras neuronales, la adquisición y el uso de las habilidades cognitivas y otros elementos, como la interacción y la dinámica familiar (Ojeda, 2012; Sans y Gozal, 2008). En el caso de los niños, se ha visto que el sueño y sus diferentes dinámicas desempeñan un papel fundamental en todos los componentes de su vida, incluidos la salud física, el rendimiento académico, la relación con sus pares y el desarrollo de los roles dentro del contexto familiar (Ojeda, 2012), aspectos todos ellos que pueden verse afectados por las diferentes condiciones que producen el confinamiento y el aislamiento social.

Se estima que la prevalencia de trastornos del sueño en todo el mundo oscila entre 35 y $45 \%$ de la población mayor de 18 años (Tlatoa, Ocaña, Márquez, Bermeo y Gallo, 2015). Otros estudios hechos sobre las dificultades del sueño y su calidad en la población infantil arrojan estadísticas similares, con signos y síntomas que tienen una alta prevalencia en muestras de todo el mundo. Entre los problemas de sueño más recurrentes se encuentran los terrores nocturnos, la cantidad insuficiente de 
horas de sueño, el sueño interrumpido y poco reparador, conductas relacionadas con el sonambulismo y la somnolencia diurna, los cuales, según algunos estudios (Fatan y Haghighi, 2018; Gupta et al., 2016; Martínez et al., 2020), pueden afectar entre 8 y $20 \%$ de los niños de 6 a 13 años de edad, encontrándose también un porcentaje considerable de menores que padecen enuresis como una dificultad relacionada directamente con las alteraciones del sueño.

En el caso de Colombia, los estudios indican que los trastornos del sueño y otros problemas relacionados como los ya mencionados muestran una prevalencia superior a $20 \%$ en poblaciones de niños y adolescentes de 5 a 17 años de edad, siendo la duración y la irregularidad en los patrones del sueño, el bruxismo y la somniloquia los trastornos más recurrentes (Ramírez et al., 2008; Vélez, Zamora, Bautista y Calderón, 2018).

Es importante señalar que hay una amplia gama de condiciones físicas y mentales que se relacionan con una inadecuada calidad del sueño y el descanso, algunas de las cuales pueden ser la causa de distintos trastornos, mientras que otras guardan una relación que aún se investiga. De lo anterior se destacan enfermedades respiratorias como la rinitis, la cual se ha comprobado que genera una interrupción continua del sueño y ronquidos (Leger, Bonnefoy, Pigearias, de La Giclais y Chartier, 2017). Asimismo, se han identificado relaciones entre la obesidad y algunas dificultades gastrointestinales -como el colon irritable-con problemas para conciliar el sueño, las que alcanzan una prevalencia superior a $30 \%$ en las muestras estudiadas (Wang, Duan y Duan, 2018; Melinda y Sekartini, 2019).

Asimismo, hay que añadir los vínculos existentes entre diferentes trastornos mentales y la afectación que generan en las dinámicas normales del sueño. Se sabe que uno de los signos más comunes de los trastornos depresivos o los de ansiedad son las alteraciones en el sueño, al igual de lo que ocurre con los diferentes niveles del trastorno del espectro autista, en el que las dinámicas del sueño y el descanso se ven afectadas (Mazurek, Dovgan, Neumeyer y Malow, 2019). En muchos casos la aparición de cambios en los hábitos de sueño se hacen evidentes al anticiparse a otros síntomas que

Instituto de Investigaciones Psicológicas - Universidad Veracruzana ISSN impreso: 1405-1109 acompañan al diagnóstico de un cierto trastorno, razón por la cual se considera que pueden ser un predictor de dichas enfermedades, las cuales son de considerable interés en la salud pública en los tiempos de confinamiento, pues un buen análisis y una mejor comprensión de las afectaciones en los hábitos del sueño puede generar procesos de detección e intervención temprana (Torre et al., 2016).

Teniendo en cuenta lo expuesto anteriormente, la presente investigación tuvo como objetivos describir las características del sueño en una muestra de niños colombianos de entre 5 y 12 años de edad, mediante el Children's Sleep Habits Questionnaire (CSHQ), e identificar la relación entre los cambios generados por el confinamiento en casa y las características del sueño en la muestra antes mencionada.

\section{MÉTODO}

\section{Participantes}

La muestra no probabilística de tipo incidental estuvo conformada por 259 padres de familia que llenaron los cuestionarios referentes a la información relacionada con sus hijos, cuyas edades oscilaban entre 5 y 12 años $(\mathrm{M}=8.56$; D.E. $=1.5)$, de los cuales $46 \%$ eran mujeres, todos ellos residentes de la ciudad de San Juan de Pasto (Colombia). Se estableció como criterio de exclusión a aquellos participantes que reportaran tomar medicamentos que pueden generar somnolencia, y de aquellos que hubiesen sido sometidos a tratamientos quirúrgicos.

\section{Instrumentos}

\section{Cuestionario socioeconómico ad hoc}

Inicialmente se presentó a los padres un cuestionario que permitió determinar su estrato socioeconómico, así como el sexo y edades de sus hijos, en el que se incluyó una pregunta referente a la toma de medicamentos por parte de estos.

Luego, se les aplicó un cuestionario elaborado por los presentes autores que consta de 15 ítems que se califican de 0 a 4 (de "nunca" a "siempre" y 
que está conformado por tres subescalas: Hábitos relacionados con la tecnología, Cambios en la emocionalidad y Hábitos diarios tras el inicio del confinamiento. Los ítems se elaboraron teniendo en cuenta las variables propuestas por Muñoz y Bianchi, 2017. Dicho cuestionario mostró buenas propiedades psicométricas, con una medida de adecuación muestral KMO de 0.89 y prueba complementaria de esfericidad de Barlett $(p<0.05)$. En la prueba de fiabilidad para las subescalas y la escala total se hallaron los siguientes coeficientes alfa de Cronbach: Hábitos relacionados con la tecnología, 0.76; Cambios en la emocionalidad, 0.82; Hábitos diarios, 0.72, y escala total, 0.90.

Después, los padres de los participantes aplicaron el Children's Sleep Habits Questionnaire (CSHQ en lo sucesivo), adaptado al español por Alfonso, Báez, Morales y González (2019), el cual se compone de 33 ítems que se califican de 1 a 3 de la siguiente manera: Habitualmente (5-7 veces por semana), 3; Algunas veces (2-4 veces por semana), 2 , y Pocas veces (0-1 vez por semana), 1. El cuestionario CSHQ se estructura con ocho subescalas: 1: Resistencia para ir a la cama (ítems $1,3,4,5$, 6 y 8); 2: Inicio del sueño (ítem 2); 3: Duración del sueño (ítems 9, 10 y 11); 4: Ansiedad del sueño (ítems 5, 7, 8 y 21); 5: Despertares nocturnos (ítems 16, 24 y 25); 6: Parasomnias (ítems 12, 13, 14, 15, 17, 22 у 23); 7: Disturbios respiratorios del sueño (ítems 18, 19 y 20), y 8: Somnolencia diurna (ítems 26, 27, 28, 29, 30, 31, 32 y 33). Los ítems $1,2,3,10,11$ y 26 se califican de manera inversa. El cuestionario validado en español (Alfonso et al., 2019) mostró adecuadas propiedades psicométricas: coeficiente $\alpha$ de Cronbach en la prueba general de 0.67 , y de 0.42 a 0.61 en las subescalas.

\section{Procedimiento}

El proceso de reclutamiento de los participantes y recolección de información se efectuó en su totalidad de manera virtual entre los meses de junio y julio de 2020. El llenado de los cuestionarios y la firma de los formatos de consentimiento informado se llevaron a cabo en el mismo formulario del software utilizado para recolectar la información
(Google Forms), siendo de 15 minutos la duración aproximada de la aplicación. La presente investigación y sus subprocesos se fundamentan en los principios éticos contenidos en el Código Deontológico y Bioético de Psicólogo en Colombia, Ley 1090 de 2006, y además en los lineamientos establecidos por la Resolución 8430 de 1993, por la cual se dictan las normas científicas, técnicas y administrativas para la investigación en salud en Colombia, especialmente en su Título II ("De la investigación en seres humanos"). En todos los casos se explicó a los responsables de los participantes el objetivo del estudio, sus riesgos y beneficios, subrayándose el carácter anónimo, confidencial y voluntario de su participación.

\section{Análisis de datos}

Los análisis estadísticos se llevaron a cabo mediante el software SPSS.23 (IBM Corp., 2015). En primer lugar, se calcularon la frecuencia, la media y la desviación estándar en las variables cuantitativas, y las frecuencias y porcentajes en las cualitativas del cuestionario sociodemográfico.

Para dar cumplimiento al primer objetivo, se calculó el promedio y la desviación estándar de los puntajes de cada subescala y de la escala total del CSHQ; asimismo, se determinó la asociación entre las variables de sexo y nivel socioeconómico con el puntaje final de la escala a través de una prueba $X^{2}$. El segundo objetivo estaba encaminado en identificar la relación entre los indicadores referentes a la percepción por parte de los padres sobre los cambios en las rutinas diarias de los niños, en el uso de la tecnología y en la emocionalidad. Mediante el CSHQ y sus subescalas se verificó una prueba de correlación rho de Spearman. El nivel de significancia adoptado en los análisis correlacionales fue de $p<0.05$. Por último, se obtuvieron los percentiles según el puntaje total del CSHQ.

\section{RESULTADOS}

En cuanto a los estadísticos descriptivos referentes a la cantidad de sueño y su inicio, se halló que los participantes iban a la cama entre las 9 y 11 de 
la noche, reportando además un promedio acumulado de sueño (sueño nocturno y siestas diurnas) de 9.2 horas, si bien $9.1 \%$ de los participantes reportaron dormir menos de 8 horas.

El análisis del cuestionario de cambios emocionales y de hábitos percibidos por los padres tras el inicio del confinamiento mostró cambios en las rutinas en $98 \%$ de los casos analizados, evidenciándose rangos amplios en los puntajes de cada categoría y final, lo cual indica que dentro del grupo había niños que habían modificado con diferentes niveles de alteración diversos aspectos de su rutina diaria o de su esfera emocional (Tabla 1).

Tabla 1. Análisis descriptivo de las subescalas del cuestionario de cambios emocionales y de hábitos percibidos tras el confinamiento $(\mathrm{N}=259)$.

\begin{tabular}{|l|c|c|c|r|}
\hline \multicolumn{1}{|c|}{ Subescala } & Mín. & Máx. & M & \multicolumn{1}{c|}{ D.E. } \\
\hline Hábitos relacionados con el uso de tecnología & 1 & 16 & 6.20 & 3.14 \\
\hline Cambios en la emocionalidad & 0 & 25 & 9.24 & 5.20 \\
\hline Hábitos diarios & 0 & 16 & 6.00 & 3.21 \\
\hline TOTAL & 3 & 56 & 21.44 & 10.50 \\
\hline
\end{tabular}

Los ítems con el más alto puntaje fueron, a saber: 1: "Utiliza celular, computador o consola de video juegos con más frecuencia” ( $\mathrm{M}=1.90)$; 6: “El niño reporta querer volver a la normalidad" $(\mathrm{M}=1.73)$; 10: "El niño consume más alimentos de lo habitual” ( $\mathrm{M}=1.49)$; 12: "El niño ha cambiado la hora de despertar, de tomar siestas y de ir a la cama" $(\mathrm{M}=1.48)$, evidenciando que los cambios no se li- mitaban a un aspecto en particular y que afectaban a los menores en diferentes ámbitos.

Para dar cumplimiento al primer objetivo del presente estudio se hicieron análisis descriptivos del CSHQ, hallándose una media de respuesta de 43.53 (D.E. $=8.96$ ), con un rango de 35 a 80 puntos, y se obtuvieron la media, el rango y la desviación estándar de las ocho subescalas de dicha prueba (Tabla 2).

Tabla 2. Análisis descriptivo de las subescalas del CSHQ $(\mathrm{N}=259)$.

\begin{tabular}{|l|r|r|r|c|}
\hline \multicolumn{1}{|c|}{ Subescala } & Mín. & \multicolumn{1}{c|}{ Máx. } & \multicolumn{1}{c|}{ M } & D.E. \\
\hline Resistencia para ir a la cama & 6 & 16 & 7.49 & 2.412 \\
\hline Inicio del sueño & 1 & 3 & 1.3 & 0.653 \\
\hline Duración del sueño & 3 & 9 & 3.68 & 1.208 \\
\hline Ansiedad del sueño & 4 & 11 & 5.22 & 1.468 \\
\hline Despertares nocturnos & 3 & 9 & 3.72 & 1.282 \\
\hline Parasomnias & 7 & 19 & 9.03 & 2.769 \\
\hline Disturbios respiratorios del sueño & 3 & 9 & 3.75 & 1.245 \\
\hline Somnolencia diurna & 8 & 21 & 9.34 & 2.331 \\
\hline TOTAL & 35 & 80 & 43.53 & 8.965 \\
\hline
\end{tabular}

Los análisis muestran promedios altos en las subescalas de Parasomnias, Somnolencia diurna y Resistencia para ir a la cama. Adicionalmente, se evidencia un rango amplio en los puntajes de las subescalas de la prueba, lo que indica la presencia de sujetos con altas calificaciones que señalan dificultades del sueño. Los puntajes del CSHQ mostraron que no había diferencias estadísticamente significativas respecto a las variables de sexo $\left(X^{2}=44.12\right.$; $p>0.10)$ y nivel socioeconómico $\left(X^{2}=131.17\right.$; $p>0.5)$. Por otra parte, se determinó la frecuencia de respuesta expresada en porcentaje, la media y la desviación estándar para cada ítem (Tabla 3 ). 
Tabla 3. Tabla de frecuencias por ítems del CSHQ.

\begin{tabular}{|c|c|c|c|c|c|c|}
\hline Núm. & Ítem & $\begin{array}{l}\text { Usualmente } \\
\quad(5-7)\end{array}$ & $\begin{array}{c}\text { Algunas } \\
\text { veces } \\
(2-4)\end{array}$ & $\begin{array}{l}\text { Rara vez } \\
(0-1)\end{array}$ & M & D.E. \\
\hline 1 & El niño va a dormir a la misma hora todas las noches. & 81.1 & 11.6 & 7.3 & 1.26 & .585 \\
\hline 2 & $\begin{array}{l}\text { El niño se duerme dentro de los } 20 \text { minutos después } \\
\text { de ir a la cama. }\end{array}$ & 81.1 & 8.1 & 10.8 & 1.30 & .653 \\
\hline 3 & El niño se duerme en su cama. & 86.1 & 8.5 & 5.4 & 1.19 & .515 \\
\hline 4 & El niño se duerme en la cama de su hermano. & 83.4 & 6.1 & 10.4 & 1.27 & .638 \\
\hline 5 & $\begin{array}{l}\text { El niño necesita de la presencia de los padres en la habitación } \\
\text { para dormirse. }\end{array}$ & 82.6 & 10.8 & 6.6 & 1.24 & .561 \\
\hline 6 & $\begin{array}{l}\text { El niño pone resistencia a la hora de dormir (llora, rechaza } \\
\text { estar en la cama). }\end{array}$ & 86.1 & 9.7 & 4.2 & 1.18 & .484 \\
\hline 7 & El niño teme dormir en la oscuridad. & 83.8 & 10.4 & 5.8 & 1.22 & .431 \\
\hline 8 & El niño teme dormir solo. & 75.3 & 15.4 & 9.3 & 1.34 & .641 \\
\hline 9 & El niño duerme muy poco. & 85.7 & 8.9 & 5.4 & 1.20 & .517 \\
\hline 10 & El niño duerme la cantidad correcta. & 77.2 & 17.4 & 5.4 & 1.28 & .558 \\
\hline 11 & El niño duerme aproximadamente la misma cantidad cada día. & 83.4 & 13.1 & 3.5 & 1.20 & .480 \\
\hline 12 & El niño se orina en la cama en la noche. & 91.5 & 1.6 & 6.9 & 1.25 & .575 \\
\hline 13 & El niño habla durante el sueño. & 92.7 & 7.3 & 0 & 1.32 & .604 \\
\hline 14 & El niño es inquieto y se mueve mucho durante el sueño. & 78.0 & 14.3 & 7.7 & 1.30 & .604 \\
\hline 15 & El niño camina durante la noche. & 80.3 & 12.7 & 6.9 & 1.27 & .579 \\
\hline 16 & $\begin{array}{l}\text { El niño se cambia a la cama de otra persona durante la noche } \\
\text { (padres, hermanos). }\end{array}$ & 86.1 & 10.4 & 3.5 & 1.17 & .462 \\
\hline 17 & $\begin{array}{l}\text { El niño rechina los dientes durante el sueño } \\
\text { (su dentista puede habérselo dicho). }\end{array}$ & 75.7 & 17.0 & 7.3 & 1.32 & .604 \\
\hline 18 & El niño ronca fuertemente. & 78.4 & 17.8 & 3.9 & 1.25 & .518 \\
\hline 19 & El niño parece detener la respiración durante el sueño. & 82.6 & 12.4 & 5 & 1.22 & .525 \\
\hline 20 & El niño se ahoga durante el sueño. & 79.2 & 14.3 & 6.6 & 1.27 & .576 \\
\hline 21 & $\begin{array}{l}\text { El niño tiene problemas para dormir fuera de su casa } \\
\text { (visita a familiares, de vacaciones). }\end{array}$ & 76.1 & 16.6 & 7.3 & 1.31 & .603 \\
\hline 22 & $\begin{array}{l}\text { El niño despierta durante la noche gritando, sudando } \\
\text { y sin consuelo. }\end{array}$ & 71.4 & 16.6 & 12.0 & 1.41 & .694 \\
\hline 23 & $\begin{array}{l}\text { El niño despierta alarmado por un sueño aterrador } \\
\text { (pesadillas). }\end{array}$ & 85.3 & 11.6 & 3.1 & 1.18 & .457 \\
\hline 24 & El niño se despierta una vez durante la noche. & 79.9 & 12.4 & 7.7 & 1.28 & .597 \\
\hline 25 & El niño se despierta más de una vez en la noche. & 79.9 & 13.1 & 6.9 & 1.27 & .581 \\
\hline 26 & El niño despierta por sí solo. & 88.4 & 6.9 & 4.6 & 1.16 & .479 \\
\hline 27 & El niño despierta de mal carácter, disgustado. & 84.6 & 8.9 & 6.6 & 1.22 & .551 \\
\hline 28 & El niño es despertado por adultos, hermanos. & 87.3 & 7.3 & 5.4 & 1.18 & .508 \\
\hline 29 & El niño tiene dificultades para salir de la cama en la mañana. & 90.0 & 6.9 & 3.1 & 1.13 & .420 \\
\hline 30 & $\begin{array}{l}\text { El niño demora mucho tiempo para estar alerta en la mañana } \\
\text { (despabilarse). }\end{array}$ & 88.4 & 9.7 & 1.9 & 1.14 & .395 \\
\hline 31 & El niño ronca durante el día. & 84.2 & 9.7 & 6.2 & 1.22 & .544 \\
\hline 32 & El niño pareció dormido o somnoliento mirando la TV. & 87.6 & 10.0 & 2.3 & 1.15 & .415 \\
\hline 33 & $\begin{array}{l}\text { El niño pareció dormido o somnoliento durante paseos } \\
\text { en un carro. }\end{array}$ & 88.8 & 8.1 & 3.1 & 1.14 & .430 \\
\hline
\end{tabular}


Como se aprecia, $17.5 \%$ de los participantes reportaron al menos una dificultad del sueño, de los cuales $6 \%$ indicó que la misma era grave o significativa. Los ítems que obtuvieron el promedio más alto fueron el 8: El niño teme dormir solo; el 13: El niño habla durante el sueño; el 17: El niño rechina los dientes durante el sueño, y el 22: El niño tiene problemas para dormir fuera de su casa.

En cuanto al segundo objetivo, se aplicó la prueba rho de Spearman para determinar las correlaciones entre los puntajes de las subescalas del CSHQ con sus similares del cuestionario de percepción sobre los cambios emocionales y en las rutinas diarias tras el confinamiento (Tabla 4), encontrándose correlaciones positivas (bajas y moderadas. Cabe mencionar que sobresalieron los siguientes factores del CSHQ: Resistencia para ir a la cama, Parasomnias y Somnolencia diurna, que arrojaron correlaciones significativas con los componentes del cuestionario de cambios emocionales y en las rutinas tras el confinamiento.

Tabla 4. Correlaciones entre las subescalas del CSHQ y la percepción sobre los cambios emocionales y las rutinas diarias tras el confinamiento.

\begin{tabular}{|c|c|c|c|c|c|c|c|c|c|}
\hline & $\begin{array}{c}\text { Resistencia } \\
\text { para ir a } \\
\text { la cama }\end{array}$ & $\begin{array}{c}\text { Inicio } \\
\text { del } \\
\text { sueño }\end{array}$ & $\begin{array}{l}\text { Duración } \\
\text { del sueño }\end{array}$ & $\begin{array}{c}\text { Ansiedad } \\
\text { en el } \\
\text { sueño }\end{array}$ & $\begin{array}{c}\text { Despertares } \\
\text { nocturnos }\end{array}$ & Parasomnias & $\begin{array}{c}\text { Disturbios } \\
\text { respiratorios } \\
\text { del sueño }\end{array}$ & $\begin{array}{c}\text { Somno- } \\
\text { lencia } \\
\text { diurna }\end{array}$ & $\begin{array}{l}\text { Total } \\
\text { CSHQ }\end{array}$ \\
\hline Uso de tecnologías & $0.23 *$ & $0.53 *$ & $0.17 *$ & 0.06 & 0.07 & 0.07 & 0.04 & $0.62 *$ & $0.87 *$ \\
\hline $\begin{array}{l}\text { Cambios } \\
\text { emocionales }\end{array}$ & $0.15^{*}$ & 0.11 & 0.06 & 0.01 & $0.61 *$ & $0.53 *$ & 0.60 & $0.74 *$ & $0.92 *$ \\
\hline Cambios en hábitos & $0.13 *$ & 0.01 & 0.02 & 0.10 & $0.24 *$ & $0.16^{*}$ & 0.75 & $0.64 *$ & $0.87^{*}$ \\
\hline Total & $0.26^{*}$ & $0.15^{*}$ & 0.97 & $0.68 *$ & 0.88 & $0.15^{*}$ & 0.07 & $0.75^{*}$ & $0.46^{*}$ \\
\hline
\end{tabular}

*Nivel de significancia $p<0.05$.

A modo de complemento, se obtuvieron los percentiles para el CSHQ con el fin de determinar los pun- tajes totales que se pueden considerar problemáticos y patológicos, lo que se muestra en la Tabla 5.

Tabla 5. Tabla de percentiles y puntaje total en el CSHQ ( $=259)$.

\begin{tabular}{|l|l|l|l|l|l|l|l|l|l|}
\hline Percentil & 10 & 20 & 30 & 40 & 50 & 60 & 70 & 80 & 90 \\
\hline Puntaje total & 35 & 36 & 37 & 39 & 41 & 42 & 46 & 50 & 55 \\
\hline Número de casos & 33 & 25 & 22 & 31 & 32 & 13 & 31 & 21 & 51 \\
\hline
\end{tabular}

\section{DISCUSIÓN}

Los resultados de este trabajo indican cambios en las rutinas de los niños, sobresaliendo el aumento del uso de aparatos tecnológicos y cambios en las rutinas alimenticias, situación que puede relacionarse con el desarrollo de los procesos académicos de manera virtual (Ministerio de Educación Nacional, 2020; sin embargo, se observa que el puntaje más alto se centra en los cambios emocionales que se manifiestan a través de un mayor consumo de alimentos (Vergara, Lobato, Díaz y Del Rosario, 2020), el deseo de volver a la normalidad, y el aburrimiento por permanecer en casa durante largos periodos de tiempo (González y Mirón, 2020).

En cuanto a los hábitos de sueño, en esta investigación se halló que la cantidad de horas de sueño eran las adecuadas para los niños $(M=9.2)$, de los que cerca de $10 \%$ reportó dormir menos horas de las recomendadas, cifra similar a la encontrada por Arboledas, Alarcón, González, Roselló y Salort (2011) en una muestra española, pero que se diferencia de la mayoría de estudios sobre esta población, en la que la insuficiencia de horas de sueño es superior, alcanzando una prevalencia de 25\% (Muñoz y Bianchi, 2017; Oropeza, López y Granados, 2019). Tal resultado puede explicarse 
debido al cambio en las dinámicas escolares, la educación virtual y la falta de continuidad en los procesos educativos, haciendo que los hábitos del sueño se asemejen a las rutinas que adoptan los niños durante los fines de semana (Muñoz y Bianchi, 2017).

En relación a lo anterior, las dificultades del sueño también sufrieron cambios tras el inicio del confinamiento. Los resultados indican que, si bien la cantidad de sueño es suficiente, se observa que $45.5 \%$ de los casos reportan dificultades del sueño, mayormente relacionadas con parasomnias, somnolencia diurna y resistencia para ir a la cama, las cuales se relacionan directamente con el cambio de rutina y el aumento en los niveles de ansiedad (Avendaño et al., 2020). En cuanto al factor específico de ansiedad del sueño, se encuentra un puntaje que sobresale ante al resto de las subescalas; sin embargo, la falta de información sobre la ansiedad en los niños durante el confinamiento no permite contrastar la información encontrada en la muestra, y se debe limitar a las proyecciones del aumento en los problemas de salud mental alegados por Huarcaya (2020) y Sandín, Valiente, García y Chorot (2020).

Complementando lo expuesto, las pruebas de $X^{2}$ evidenciaron que no había diferencias estadísticamente significativas entre los puntajes del CSHQ y las variables de sexo y de nivel socioeconómico, resultados similares a los encontrados por Arboledas et al. (2011), quienes afirman que dichas variables no son determinantes en las dificultades del sueño, a diferencia de la edad o la etapa del desarrollo, las cuales han mostrado ser buenos predictores de dificultades tales como la cantidad de sueño o la hora de ir a la cama, lo que debe tenerse en cuenta para próximos estudios en los cuales se consideren rangos de edad más amplios o se utilicen muestras compuestas por niños, preadolescentes y adolescentes.

Al determinar el nivel de relación entre los problemas del sueño y las dinámicas del confinamiento generadas por la pandemia de Covid-19, se encontró que los problemas de mayor prevalencia en los análisis descriptivos mostraron también correlaciones estadísticamente significativas con las subescalas del cuestionario de cambios de hábitos y emociones. En este orden de ideas, la somnolencia diurna es el factor del CSHQ que mayores correlaciones arrojó, siendo este un problema central en el presente estudio, cuyos resultados son similares a la evidencia científica hallada, que señala que esa somnolencia es producto de un uso excesivo de aparatos tecnológicos (Aguilar, Vergara, Velásquez y García, 2015), y además de la ausencia de hábitos estables y adecuados al momento de ir a la cama (Lopes et al., 2016), constituyendo así un signo característico de los cuadros ansioso-depresivos (Andreu y Vicario, 2010). De igual manera, se destaca una correlación moderada entre el factor Parasomnias del CSHQ con la subescala de cambios emocionales, que muestra una prevalencia mayor en los ítems que evalúan la inquietud durante la noche, los terrores nocturnos y el bruxismo, signos estos que se vinculan con afectaciones emocionales y niveles elevados de ansiedad (Firmani et al., 2015; Miró et al., 2005; Orgilés, Fernández, Gonzálvez y Espada, 2016).

Por otra parte, los resultados obtenidos tras el cálculo de percentiles permiten considerar que las personas con puntuaciones menores a las halladas en el percentil 50 (41 puntos) tienen un riesgo bajo de verse afectados en su calidad de sueño y descanso o de manifestar alguna afectación patológica del sueño, mientras que los puntajes superiores indican patologías del sueño (Owens, Spirito y McGuinn, 2000). Cabe señalar que algunas investigaciones psicométricas plantean que puntuaciones mayores o iguales al percentil 85 (50 puntos) indican un riesgo de afectación moderado o la presencia de signos patológicos, y que los puntajes mayores o iguales al percentil 95 (55 puntos) representan un problema de salud inminente que requiere atención inmediata (Peris, Maganto y Garaigordobil, 2018).

Por todo lo anterior, se considera indispensable realizar estudios sobre los factores protectores de la calidad del sueño y de la salud en general para afrontar la pandemia (por ejemplo, ejercicio físico y uso adecuado de aparatos tecnológicos).

Debido a que el presente estudio abordó un problema nuevo e incidental, se recomienda analizar a profundidad la relación de variables como las alteraciones del sueño y de signos y síntomas de ansiedad con el uso inadecuado de aparatos tecnológicos, para así determinar de un modo más pre- 
ciso si el abuso tecnológico es una manifestación ansiosa, o bien obedece a otras condiciones, por ejemplo el aburrimiento generado por el entorno.

Es recomendable realizar más investigaciones sobre los problemas del sueño durante la crisis sanitaria generada por el Covid-19 con muestras más heterogéneas y una mayor cantidad de análisis transculturales para determinar con mayor profundidad el impacto de los cambios generados por esta pandemia en los hábitos de sueño de las personas.
Las limitaciones de este trabajo están relacionadas con el carácter transversal de su diseño metodológico, lo que puede restringir las inferencias relacionales y limitar la interpretación de los resultados obtenidos. Se reconoce que es necesario a futuro corroborar los resultados mediante estudios longitudinales; por otra parte, se recomienda realizar estudios que analicen diferentes variables relacionadas con la salud de los niños y adolescentes durante el confinamiento por Covid-19 en virtud de que la información disponible para realizar contrastes es sumamente limitada.

Citación: Rosero B., A.D y Ortega E., G.E. (2022). Hábitos de sueño en el confinamiento por Covid-19 en una muestra de niños colombianos. Psicología y Salud, 32(1), 125-135. https:// Doi.org/10.25009/pys.v32i1.2719.

\section{REFERENCIAS}

Aguilar M., M., Vergara F., A., Velásquez E., J. y García H., A. (2015). Actividad física, tiempo de pantalla y patrones de sueño en niñas chilenas. Anales de Pediatría, 83(5), 304-310.

Alfonso M., B., Báez M., M., Morales C., L. y González J., E. (2019). Validación al español de un cuestionario de hábitos de sueño en los niños. Revista Cubana de Pediatría, 91(2). Recuperado de http://scielo.sld.cu/scielo.php?script=sci_arttextypid $=$ S00345312019000200011ylng=esytlng=pt.

Andreu M., M. y Vicario M., H. (2010). Hipersomnia. Somnolencia diurna excesiva y alteraciones del ritmo circadiano en pediatría. Pediatría Integral, 720.

Arboledas G., P., Alarcón M., C., González G., M., Roselló A., L. y Salort M., M. (2011). Hábitos y problemas con el sueño de los 6 a los 14 años en la Comunidad Valenciana. Opinión de los propios niños. Anales de Pediatría, 74(2) $103-115$.

Avendaño, L., Cerda, S., Giadach, C.G., Hernández, A., León, P., Menéndez, P., Mesa, T., Ortega, P. y Riffo, C. (2020). Recomendaciones para un buen dormir durante cuarentena por COVID-19. Revista Chilena de Pediatría, 91(7), 1.

Fatan, F. y Haghighi, M. (2018). Sleep pattern evaluation of 8 to 12-year-old students in Shiraz. Journal of Comprehensive Pediatrics, 9(2). Recuperado de https://Doi.org/10.5812/compreped.58289.

Ferrer, R. (2020). Pandemia por Covid-19: el mayor reto de la historia del intensivismo. Medicina Intensiva, 44(6), $323-324$.

Firmani, M., Reyes, M., Becerra, N., Flores, G., Weitzman, M. y Espinosa, P. (2015). Bruxismo de sueño en niños y adolescentes. Revista Chilena de Pediatría, 86(5), 373-379.

Girona, T. (2020). Tiempo de confinamiento requerido para evitar un rápido rebote de COVID-19: predicciones de un modelo estocástico de Monte Carlo. Frontiers in Physics, 8, 186.

González C., P. y Mirón G., R. (2020). "No te conozco, pero aquí estoy”: Cartas solidarias en tiempos de COVID-19 en España. Revista Española de Comunicación en Salud, Julio, 226-233. Doi: 10.20318/recs.2020.5425.

González-Jaramillo, V., González J., N., Gómez R., C., Palacio A., C.A., Gómez L., A. y Franco O., H. (2020). Proyecciones de impacto de la pandemia COVID-19 en la población colombiana, según medidas de mitigación. Datos preliminares de modelos epidemiológicos para el periodo del 18 de marzo al 18 de abril de 2020. Revista de Salud Pública, 22(1).

Gupta, R., Goel, D., Kandpal, S.D., Mittal, N., Dhyani, M. y Mittal, M. (2016). Prevalence of sleeping disturbances in elementary school children. The Indian Journal of Pediatrics, 83(11), 1232-1236.

Huarcaya V., J. (2020). Consideraciones sobre la salud mental en la pandemia de COVID-19. Revista Peruana de Medicina Experimental y Salud Pública, 37(2), 327-334. Doi: 10.17843/rpmesp.2020.372.5419.

IBM Corporation (2015). IBM SPSS Statistics for Windows, Version 23.0. Armonk, NY: IBM Corp.

Leger, D., Bonnefoy, B., Pigearias, B., De la Giclais, B. y Chartier, A. (2017). Poor sleep is highly associated with house dust mite allergic rhinitis in adults and children. Allergy, Asthma \& Clinical Immunology, 13(1), 36. Doi: 10.1186/s13223-0170208-7.

Lopes, S., Almeida, F., Jacob, S., Figueiredo, M., Vieira, C. y Carvalho, F. (2016). "Diz-me como dormes": hábitos e problemas de sono em crianças portuguesas em idade pré-escolar e escolar. Nascer e Crescer, 25(4), 211-216. 
Martínez C., L., March V., J.A., Valencia G., V., Serrano D., A., Conca B., M.Á., Polo R., A., Domínguez H., C. y Boronat T., F. (2020). Evaluación de la higiene del sueño y la prevalencia de los trastornos del sueño en pacientes con enuresis monosintomática. Utilidad de la herramienta de detección de sueño BEARS. Actas Urológicas Españolas, 44(7), 477-482.

Mazurek, M.O., Dovgan, K., Neumeyer, A.M. y Malow, B.A. (2019). Course and predictors of sleep and co-occurring problems in children with autism spectrum disorder. Journal of Autism and Developmental Disorders, 49(5), 2101-2115. Doi: 10.1007/s10803-019-03894-5.

Mazza, C., Ricci, E., Biondi, S., Colasanti, M., Ferracuti, S., Napoli, C. y Roma, P. (2020). A nationwide survey of psychological distress among Italian people during the COVID-19 pandemic: Immediate psychological responses and associated factors. International Journal of Environmental Research and Public Health, 17(9), 3165. Recuperado de https://Doi.org/10.3390/ ijerph17093165.

Melinda, T.F. y Sekartini, R. (2019). Association between obesity and sleep disorders in primary school children: a cross-sectional study. Medical Journal of Indonesia, 28(2), 167-173. Doi: 10.13181/mji.v28i2.2645.

Mera A., Y., Tabares G., E., Montoya G., S., Muñoz R., D.I. y Vélez F., M. (2020). Recomendaciones prácticas para evitar el desacondicionamiento físico durante el confinamiento por pandemia asociada a COVID -19. Universidad y Salud, 22(2), 166-177. Doi: 10.22267/rus.202202.188.

Ministerio de Educación Nacional (2020). Orientaciones para el manejo de la emergencia por Covid 19 por parte de los establecimientos educativos privados. Marzo 15. Bogotá: Autor. Recuperado de https://www.mineducacion.gov.co/1759/ articles-394243_recurso_1.pdf-.

Miró, E., Lozano M., D. y Casal G., B. (2005). Sueño y calidad de vida. Revista Colombiana de Psicología, 14, 11-27.

Muñoz Q., A. y Bianchi S., B. (2017). Hábitos de sueño, desempeño académico y comportamiento en niños de básica primaria. Pensando Psicología, 13(21), 5-17.

Ojeda del V., M. (2012). El sueño en la edad preescolar y su repercusión en el desarrollo, la conducta y el aprendizaje. Revista Cubana de Higiene y Epidemiología, 50(2), 198-204.

Organización Panamericana de la Salud [OPS] (2020). Consideraciones de la Organización Panamericana de la Salud con respecto a la propagación del nuevo coronavirus emergente. Washington D.C: OPS.

Orgilés, M., Fernández M., I., Gonzálvez M., T. y Espada J., P. (2016). Niños con síntomas de ansiedad por separación: un estudio de sus hábitos y problemas de sueño. Ansiedad y Estrés, 22(2-3), 91-96.

Oropeza B., G., López S., J.D. y Granados R., D.E. (2019). Hábitos de sueño, memoria y atención en niños escolares. Revista Mexicana de Neurociencias, 20(1), 42-49.

Owens, J.A., Spirito, A. y McGuinn, M. (2000). The Children's Sleep Habits Questionnaire (CSHQ): psychometric properties of a survey instrument for school-aged children. Sleep, 23(8), 1043-1052. Doi: 10.1093/sleep/23.8.1d.

Peris, M., Maganto, C. y Garaigordobil, M. (2018). Escala de Riesgo de Adicción Adolescente a las Redes Sociales e Internet: fiabilidad y validez. Revista de Psicología Clínica con Niños y Adolescentes, 5(2), 30-36.

Ramírez M., M., Martínez L., C., Velásquez M., C., Peña Á., M., Carmona J., L. y Ochoa W., C. (2008). Prevalencia de los trastornos del sueño en niños escolares de Sabaneta, Colombia, 2005. Iatreia, 21(2), 113-120.

Sandín, B., Valiente, R.M., García E., J. y Chorot, P. (2020). Impacto psicológico de la pandemia de Covid-19: Efectos negativos y positivos en población española asociados al periodo de confinamiento nacional. Revista de Psicopatología y Psicología Clínica, 25(1), 1-22.

Sans C., O. y Gozal, D. (2008). Consecuencias neurobiológicas del síndrome de apnea del sueño infantil. Revista de Neurología, 47(12), 659-664.

Stanley, I.H., Hom, M.A., Luby, J.L., Joshi, P.T., Wagner, K.D., Emslie, G.J., Walkup, J.T., Axelon, D.A. y Joiner, T.E. (2017). Comorbid sleep disorders and suicide risk among children and adolescents with bipolar disorder. Journal of Psychiatric Research, 95, 54-59. Doi: 10.1016/j.jpsychires.2017.07.027.

Tlatoa H., M., Ocaña H., L., Márquez M., L., Bermeo, J. y Gallo A., F. (2015). El género, un factor determinante en el riesgo de somnolencia. Revista de Medicina e Investigación, 3(1), 17-21.

Torre B., L., Fernández P., R., Martínez B., D., Pérez P., R., Guzmán B., A. y García S., M. (2016). Los síntomas de sueño son predictores del número de comorbilidades. Estudio poblacional. Neumología y Cirugía de Tórax, 75(3), 209-214.

Urzúa, A., Vera V., P., Caqueo U., A. y Polanco C., R. (2020). La Psicología en la prevención y manejo del coviD-19. Aportes desde la evidencia inicial. Terapia psicológica, 38(1), 103-118. https://Doi.org/10.4067/S0718-48082020000100103.

Vargas C., M., Acosta R., G. y Bernilla A., T. (2020). El nuevo coronavirus y la pandemia del Covid-19. Revista Médica Herediana, 31(2), 125-131.

Vélez R., R., Zamora L., H., Bautista J., E. y Calderón E., G. (2018). Confiabilidad y validez del cuestionario de trastornos de sueño BEARS en niños y adolescentes escolares de Bogotá, DC, Colombia: Estudio FUPRECOL. Retos: Nuevas Tendencias en Educación Física, Deporte y Recreación, 34, 89-93. 
Vergara C., A., Lobato L., M.F., Díaz G., M. y Del Rosario A., M. (2020). Cambios en el comportamiento alimentario en la era del COVID-19. Revista Latinoamericana de Investigación Social, 3(1), 27-30.

Wang, B., Duan, R. y Duan, L. (2018). Prevalence of sleep disorder in irritable bowel syndrome: a systematic review with meta-analysis. Saudi Journal of Gastroenterology, 24(3), 141. Doi: 10.4103/sjg.SJG_603_17.

Zhou, S.J., Zhang, L.G., Wang, L.L., Guo, Z.C., Wang, J.Q., Chen, J.C. y Chen, J.X. (2020). Prevalence and socio-demographic correlates of psychological health problems in Chinese adolescents during the outbreak of COVID-19. European Child \& Adolescent Psychiatry, 29, 749-758. Doi: 10.1007/s00787-020-01541-4. 